\title{
KLAGE DER FRAU, BOTSCHAFT DES GEMAHLS UND RUINE.
}

Im Exeterbuche stehen unter den rätseln einige lyrische gedichte, uber die sich allmählich durch Conybeare, Thorpe, Ettmiller und Grein eine feststehende meinung gebildet hat, so dass dieselben in den literaturgeschichten angefuhrt werden als besonders geeignete beispiele, um die elegische dichtung der Angelsachsen zu erläutern, und dass die gelehrten unter einander dartuber verhandeln, wie die auffassung, uber die sie im ganzen einig sind, im einzelnen zu gestalten sei. Ich will und kann nichts dazu beitragen, diesen streit zu entscheiden, sondern werde im gegenteil zeigen, auf wie unsicherer grundlage die landliufige gesammtauffassung dieser stticke beruht. (Ueber das bisher für dieselben weleistete vergleiche die zusammenstellung bei Wulker, Grundriss d. ags. Litt. s. $211 \mathrm{ff}$., $224 \mathrm{ff}$.).

Von dem in der handschrift blatt $123^{a}{ }^{\mathrm{b}}$ stehenden gedichte, das seit Grein als eines gelesen und 'botschaft des gemahls' genannt wird, sagte Wuilker, Anglia II, s. 381: 'An dieses gedicht kniipfen sich keine weiteren literarischen fragen'. Ich denke, frage genug sind die runen gegen das ende, die man nicht einmal sicher lesen kann und die zu deuten, so viel ich weiss, man bis jetzt noch nicht einmal versucht hat. Auch wie man die worte gecyre in cetsomne $S R$ geador E.A $W^{\prime}$ ond $D$ (oder $M$ ) äpe benemnon zu emendieren, konstruieren oder aufzufassen habe, ist meines wissens bisher nicht gefragt worden. gecyre ist kein englisches wort (Schipper's genyre naturlich erst recht nicht).

Inzwischen hat man auch schon in der Bukowina literarische fragen an dieses gedicht gekntipft. Strobl hat in Haupt's zs. XXXI, s. 55,56 behauptet, aber nicht begründet, dass dieses gedicht auflösung des vorangehenden 61. rätsels sei, welches 
Dietrich falsch aufgefasst habe. Diese beiden sttucke, rätsel 61 und botschaft, zusammen sollen ein beispiel sein fur altenglische wechsellieder oder wettgedichte, deren vorbandensein Strobl dort nachzuweisen sucht. Ich muss gestehen, eine lange auflösung für ein ziemlich kurzes rätsel! Noch sonderbarer ist, dass Strobl mit keinem worte angeben kann, was denn nun der gegenstand des rätsels und seiner langatmigen auflösung ist. Die volkstiumliche auflösung eines rätsels besteht in der regel darin, dass die einzelnen sätze des rätsels der reihè nach vorgenommen und zug um zug ausgedeutet werden (vgl. z. b. Haupt's zs. III, s. 28, nr. 3). Davon ist hier keine rede. Dietrich's lösung des 61. rätsels ist richtig. Was Strobl mit vollem rechte an ihr tadelt, ist längst in ordnung gebracht von Muller, programm des Cöthener gymnasiums 1861, s. 18.

Dagegen halte ich es für möglich, dass dieses gedicht ein rätsel ist, wenn es auch in stil und ton und vielleicht in der art des gegenstandes von den übrigen rätseln einigermassen abweicht. Die rätsel des Exeterbuches bieten eine solche mannigfaltigkeit der gegenstände und der behandlung und so vielfach stlucke, die in ihrer art vereinzelt dastehen, dass auch die sogenannte 'botschaft des gemahls' ein rätsel einer sonst nicht vertretenen gattung sein könnte. Vielleicht ist irgend eine sache zu raten aufgegeben, die im Altenglischen durch ein wort männlichen geschlechts bezeichnet wird. Das ist die weise und das verdienst dieser altenglischen rätsel, dass 'der gegenstand des rätsels, sei er der elementaren natur oder der belebten' oder der 'durch menschenhand umgeschaffenen angehörig, erzählend auftritt, und er selbst oder der dichter in seinem namen uns von seiner heimat, von vater und mutter, von bruder und schwester, von seinen schicksalen nach vertreibung aus der heimat, von seinen taten und künsten, von kämpfen und arbeiten, von lust und leid in lebendiger schilderung berichtet' (Dietrich, Haupt's zs. XI, s. 448). Ich leugne nicht, dass die vermenschlichung des gegenstandes, wenn ein unpersönlicher gemeint ist, hier viel kräftiger als in allen anderen rätseln, beinahe hart durchgefuhrt wird. Aber der dichter konnte sich das bier erlauben, da das richtige erraten ja durch die nennung des gegenstandes in runen gesichert war. $\mathrm{Zu}$ trécynn v. 2, das Grein (der von ald.. in v. 3 noch nichts wusste), Bibl. IV, s. 551 nur zweifelnd für runenstab erklärte, 
passt vielleicht das ald .. in v. 3 , wenn es äled, feuer, oder eine form davon ist. Dann mag der gegenstand des rätsels in irgend einer weise holz oder baum sein. Freilich kann ja reld .. auch alter oder menschen sein.

Uebrigens kann in diesem stlucke ja auch ein den zuhörern des dichters bekannter mensch zu raten aufgegeben sein, wie Cynewulf im ersten rätsel. Die runen am ende ergeben, von hinten gelesen (wenn die letzte $D$ ist), dwears, also ein wort, das lautlich möglich ist in altenglischer sprache, wenn es auch nicht vorkommt, so weit sich nach den wörterblichern urteilen liisst. Es könnte sich zu ae. dweorh, an. dvergr hinsichtlich des ablautes und des suffixes ähnlich verhalten wie niederländisch niederdeutsch dwars, dwass zu ae. pweorh, mhd. twër, nhd.quer und zwerch. Die beiden wortpaare würden sich durch den wurzelanlaut unterscheiden: das erste hat germ. $d$, das zweite germ. $b$; und durch den suffixanlant: ae.dweorh, gen.dweorges hat $g$, ae. pweorh, gen. pweores hat $h$. Aber gemeinsam wäre, dass das eine mal vokal urgerm. $\ddot{e}$ mit einem guttural anlautenden suffixe, das andere mal vokal urgerm. $a$ mit einem mit $s$ anlautenden suffixe zusammentrifft. Die gutturalen suffixe können sogar ursprunglich identisch, erst im Germanischen verschiedeu geworden sein: arisches $k$ musste germanisch wegen wechselnder betonung bald $h$ geben, bald $z$ (ich meine tönendes ch oder spirantisches $y$; am besten wurde man den laut nach der analogie von $\delta$ durch ein durchstrichenes $g$ widergeben). Dieser konsonantablaut musste dann ausgeglichen werden und mochte verschieden ausgeglichen werden in verschiedenen wörtern. Das ae. *dweurs könnte also zu recht bestehen und ein männlicher eigenname sein. - Dass die runen hier so zu lesen sind wie in rätsel 20 und 74 (nach Grein's zählung), wird vielleicht gesagt durch die worte gecyrre ic atsomne, wenn so zu lesen ist, und durch geador. Rätsel 24 wird so von buchstaben gesagt: Agof is mīn namu eft onhnyrfed. Atsomne und geador wirden besagen, dass die runen nicht auseinandergerissen und ganz neu geordnet werden sollen (wie rä. 25 higora, 32 hana und hen), sondern in ihrer reihenfolge belassen, nur von hinten gelesen werden sollen. Vielleicht ist es bemerkenswert, dass hier wie rä. 20 v. 1 die runen $S$ und $R$ am anfange zusammenstehen, die dort den ausgang von hors bilden. - Doch könnten auch wie rätsel 59 und 65 das wort oder die worte, die auf- 
gegeben werden, durch runen nicht vollständig genannt, sondern nur angedeutet sein. Dann kann man in's unendliche raten. Oder ist eine oder sind einige der runen als worte aufzufassen, wie ganz gewöhnlich $W$ für wēn oder wealdend steht? Wenn $S$ den wert sige hat, bekämen wir Sigeveard. Oder liegt ein mit $d a g$, mon, $\overline{e a} d$ oder $-r \bar{e} d$ zusammengesetzter name vor? Gehörte der der geschichte oder heldensage an? - Wenn Ettmuller und Grein recht haben mit ihrer auffassung des gedichts, dass es eine botschaft des in der ferne weilenden gemahls an seine frau ist, dann kann nicht gut der name des gemahls in den runen stecken. Denn den kann die frau doch nicht vergessen haben, sie mag noch so lange von ihrem manne getrennt gewesen sein. Kurz, ich weiss mit der botschaft nichts anzufangen und muss es andern tuberlassen zu beweisen, dass sie ein rätsel ist oder nicht ist.

Ebenso steht es mit dem in der hs. folgenden gedichte, dem Conybeare den unverständlichen titel The Ruined Wallstone gab, was Thorpe änderte in das romantisch klingende The Ruin. Der anfang dieses gedichtes klingt ganz wie der anfang eines rätsels: Wrathic is paes weal-stān. Nebenbei bemerke ich: weal-stān ist auf jeden fall nicht die genaue schreibung des wortes. Grein, Bibl. IV, 673 nimmt an wie Conybeare, es stehe für weall-stan, das er zweimal belegt. Doch kann man die möglichkeit im auge behalten, dass es für wealh-stān steht, wie wealstōd neben weal/sstōd vorkommt. Nicht unmöglich scheint mir, dass hier ein ausländischer, dem keltisch-romanischen lande angehöriger stein gemeint sei. - Die letzten lesbaren worte des gedichtes sind: boet is cynelic ping huse. Ein wort pinghus wird es schwerlich gegeben haben. Die worte bat is cynelīc ping werden doch wol so zusammen gehören, nicht durch interpunktion getrennt sein. Sie klingen ganz wie aus der schlussformel eines rätsels genommen. Und was zwischen anfang und schluss steht, ist an sich so dunkel und dazu durch die uberlieferung so zerruttet, dass es ebenso wol ein rätsel als eine elegie sein zu können scheint. In v. 24 steht eine rune; der reim zeigt, dass er hier $M$ ist, nicht $D$. Sie kann ja, wie sie bisher aufgefasst worden ist, bloss eine abgekturzte schreibung für das wort mun sein; kann aber auch die letzte von den runen sein, die das rïtselwort nannten und dabei mit ihrer wortbedeutung in den text verwoben waren, 
wie die des namen Cynewulf in der Elene und im Crist. Die tibrigen runen können in den litcken v. 12-19 gestanden haben. Wenn wirklich dieses gedicht eine stadt oder burg meint, kann dieses $m$ der letzte buchstabe ihres namen gewesen sein; ortsnamen sind ja locative und zwar meistens pluralis auf -um. Uebrigens ist es augenscheinlich, dass dieses gedicht, auch abgesehen von den lücken, in der uberlieferung grosse schäden erlitten hat.

Sind die Botschaft und die Ruine rätsel, dann stehen die rätsel in der hs. nicht in drei, sondern in zwei gruppen, die zweite blatt $122-130^{\mathrm{b}}$. Das ist nicht ausser acht zu lassen, wenn man, wie schon manche versucht haben, sich eine ansicht bilden will tuber die art, wie die rätselsammlungen des Exeterbuches zu stande gekommen sind.

Ebenso wie Botschaft und Ruine steht den rätseln in ton und haltung einigermassen fern Conybeare's und Thorpe's Exile's Complaint, Grein's 'klage der frau'. Gleichwol muss ich auch bei diesem sehr dunkeln gedichte fragen, ob es nicht ein rätsel ist. Erstens scheint nämlich mir im gegensatze zu andern forschern unzweifelhaft, dass die klage und die botschaft sich auf einander beziehen, trotz den verschiedenen situationen, die sie voraussetzen; denn die gleichheit der gebrauchten ausdrilcke kann kaum zufällig sein. Die beurteilung des einen hängt von der des andern ab. Zweitens ist es gewiss bedeutsam, dass, wenn auch die klage ein rätsel ist, die rätsel in der hs. durch weiter nichts als eine sammlung geistlicher gedichte unterbrochen werden. Die vostellung, die wir uns von der geschichte der entstehung des Exeterbuches zu bilden hätten, würde dadurch sehr vereinfacht werden.

Man sieht, ich rege nur zweifel an und weise auf möglichkeiten hin, für die eintreten zu wollen mir fern liegt. Aber zwei grundsätze möchte ich feststellen, die bei der behandlung dieser drei gedichte massgebend sein sollten:

Notwendig ist eine vollständige, zusammenhängende bearbeitung dieser gedichte. Bisher hat jeder gelehrte nach dem allgemeinen eindrucke, den ihm eins derselben machte, eine 'ansicht' darüber aufgestellt und zur begrundung einzelne aus dem zusammenhange gerissene zeilen oder gar nur worte angeftihrt. Der nachfolger fand dann im texte einzelne andere ausdrlicke, die zur meinung seines vorgängers nicht stimmten, 
und begrtindete darauf eine andere 'ansicht'. - Wie mit der auffassung des inhalts ist es mit der textkritik: jeder billigte oder verwarf die emendationen des vorgängers, ohne sich zur aufgabe zu machen, einen völlig befriedigenden text hërzustellen. Seit Thorpe liest man kl. v. 20 hycgendne, während es unmethodisch ist, eine änderung hier vorzunehmen, die das folgende blīpe gebōéro in der luft schweben lässt. Die verderbniss steckt tiefer. Emendiert werden darf nur auf grund einer ganz eingehenden interpretation, die allen einzelheiten in einer befriedigenden, mit sich selbst ubereinstimmenden weise gerecht wird, und mit der die lesung and deutung der runen und die ausftullung der litcken, so weit sie möglich ist, hand in hand gehen muss. Als allerletztes ergebniss wird sich dann die literaturgeschichtliche würdigung, die man bis jetzt immer vorweggenommen hat, von selber einfinden.

So lange diese arbeit nicht geleistet ist, ist es rätlich, so wenig als möglich vorzugreifen, damit das urteil des lesers nicht befangen werde. Man lasse im abdruck diese gedichte da, wo sie in der hs. stehen: unter den rätseln. Man hält sich $\mathrm{ja}$ in andern dingen jetzt enger an die therlieferung als fruher: man setzt keine lïngezeichen, man interpungiert so wenig als möglich, man druckt den Crist und die Elene, die doch wol beide von Cynewulf sind, in verschiedenen bänden, weil der eine im Exoniensis, die andere im Vercellensis steht. Da gebe man der tuberlieferung auch die ehre bei solchen gedichten, in denen nicht weniger als alles unverstïndlich und zweifelhaft ist. Conybeare hat zwei dieser stucke aus der hs. ausgehoben und mit ahnungsreichen titeln geschmitckt, beim dritten hat das letztere Ettmuller besorgt. Niemand wird die grossen verdienste dieser beiden männer verkennen; aber ebenso wenig wird sich jemand ihnen unbedingt hingeben wollen. Und doch ist ihre meinung mit gerin wfugigen abänderungen bis heute von allen widerholt worden.

Ich schlage also vor, dass man die untersuchung unserer drei gedichte wider ganz von neuem beginne und dabei die möglichkeit im auge behalte, dass es rätsel sind.

BERLIN.

F. HICKETIER. 